Sarah Johannesmann*, Leander Claes and Bernd Henning

\title{
Lamb wave based approach to the determination of elastic and viscoelastic material parameters
}

\author{
Lamb-Wellen-basierter Ansatz zur Bestimmung elastischer und viskoelastischer Materialparameter
}

DOI 10.1515/teme-2021-0070

\begin{abstract}
In this paper a measurement procedure is presented to identify both elastic and viscoelastic material parameters of plate-like samples using broadband ultrasonic waves. These Lamb waves are excited via the thermoelastic effect using laser radiation and detected by a piezoelectric transducer. The resulting measurement data is transformed to yield information about multiple propagating Lamb waves as well as their attenuation. These results are compared to simulation results in an inverse procedure to identify the parameters of an elastic and a viscoelastic material model.
\end{abstract}

Keywords: ultrasound, inverse procedure, material parameter identification

Zusammenfassung: In diesem Beitrag wird ein Messverfahren vorgestellt, mit dem sowohl elastische als auch viskoelastische Materialparameter von plattenförmigen Proben unter Verwendung von breitbandigen Ultraschallwellen identifiziert werden können. Diese Lamb-Wellen werden über den thermoelastischen Effekt mittels Laserstrahlung angeregt und von einem piezoelektrischen Schallwandler erfasst. Die resultierenden Messdaten werden transformiert, um Informationen über sich ausbreitende Lamb-Wellen sowie deren Dämpfung zu erhalten. Die Ergebnisse werden in einem inversen Verfahren mit Simulationsergebnissen verglichen, um die Parameter eines elastischen und eines viskoelastischen Materialmodells zu identifizieren.

Schlüsselwörter: Ultraschall, inverses Verfahren, Materialparameteridentifikation

\footnotetext{
*Corresponding author: Sarah Johannesmann, Measurement Engineering Group, Paderborn University, Paderborn, Germany, e-mail: johannesmann@emt.uni-paderborn.de

Leander Claes, Bernd Henning, Measurement Engineering Group, Paderborn University, Paderborn, Germany e-Mail: claes@emt.unipaderborn.de, henning@emt.uni-paderborn.de
}

\section{Introduction}

Design processes for acoustic and ultrasonic components become increasingly simulation driven, while accurate and reliable simulations of acoustic processes require material parameters that represent the materials' dynamic behaviour. Data sheets for the applied materials, however, often provide incomplete data determined by tensile tests. Even if the datasets are complete, they only describe the quasi-static material behaviour due to the nature of the test method. As acoustic applications will expose the material to dynamic loads, the material model needs to account for frequency dependent material properties. Especially polymers exhibit viscoelastic behaviour that results in absorption of acoustic waves and needs to be considered. Viscoelastic material modelling can also be applied in ultrasonic non-destructive testing methods. As these methods also constitute examinations that apply dynamic loads, the results usually differ from destructive, quasi-static tests. Since plate-like specimens are easy to produce, evaluation methods based on Lamb waves are widespread in ultrasonic material characterisation $[6,7,11]$. The utilized experimental setups are often similar, although different methods are applied to excite and detect ultrasonic Lamb waves while varying the propagation distance in equidistant steps. The resulting spatiotemporal data is transformed using a two-dimensional Fourier transform to observe the dispersion characteristics of the Lamb waves [1]. Based on the transformed data the input parameters of a forward model, often based on a semi-analytical finite element model [3] or numerical root finding of the Rayleigh-Lamb equation [8], are varied until the computed dispersion curves match with the measured data. However, most works aim to determine only elastic material parameters while neglecting the material's absorption and viscoelastic behaviour. 


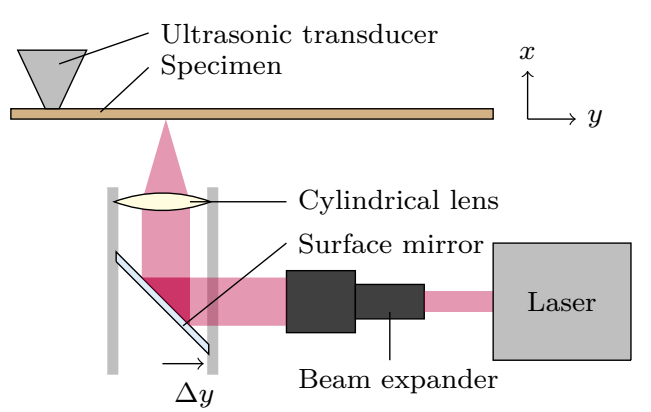

Fig. 1: Experimental setup for excitation and detection of ultrasonic Lamb waves.

\section{Experimental data acquisition and processing}

For the excitation and detection of ultrasonic Lamb waves the setup in Figure 1 is used. Short pulses (1.45 ns) of laser radiation are focused line-shaped on the specimens surface by a cylindrical lens, placed on a linear actuator to allow reproducible excitation at different positions. The sudden heating of the specimen's surface leads to excitation of broadband ultrasonic waves due to the thermoacoustic effect [5]. The ultrasonic Lamb waves are received by a purpose-built piezoceramic strip transducer [8]. Excitation at multiple equidistant positions and recording the received signals lead to a spatio-temporal signal matrix. In the absolute value of the two-dimensional Fourier transform of the matrix the propagating modes are visible as ridges. In this depiction in the frequency-wavenumber domain high intensities indicate high amplitude of the respective mode. For better visibility of each mode, some signal processing steps are performed before and after the Fourier transform, such as applying a Tukey window in time and space domain and gamma correction [12]. As an example, Figure 2 shows the result, depicting the propagating Lamb waves in a plate made of polyether ether ketone (PEEK) with a thickness of $5.45 \mathrm{~mm}$.

\subsection{Modal attenuation}

The modal acoustic attenuation is determined by subdividing the signal matrix along the spatial direction in $N$ subdatasets with a mean propagation distance $y_{n}$. Figure 3 shows this division schematically for $N=4$. Each subdataset is Fourier transformed. For each frequencyreal wavenumber pair in the subdatasets an exponential function

$$
U\left[\omega, \operatorname{Re}(k), y_{n}\right]=U_{0}[\omega, \operatorname{Re}(k)] \mathrm{e}^{\alpha[\omega, \operatorname{Re}(k)] y_{n}}
$$

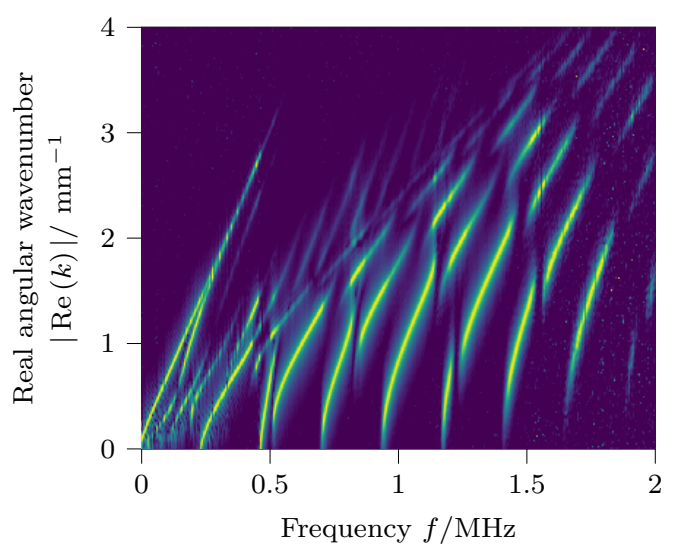

Fig. 2: Measurement result for propagating Lamb waves in a PEEK plate.

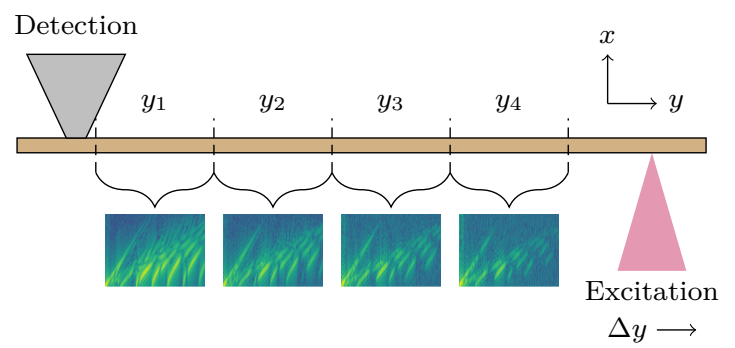

Fig. 3: Illustration of the subdivision of the dataset along the propagation distance

is fitted using the resulting intensity values $U\left[\omega, \operatorname{Re}(k), y_{n}\right]$ and the propagation distance $y_{n}$ to determine the attenuation coefficient $\alpha[\omega, \operatorname{Re}(k)]$. Plotting the attenuation coefficient over frequency and real wavenumber yields a depiction of the modal attenuation. For Figure 4 such a depiction is filtered using a rectangular mask around the theoretical propagable modes to get a better visualization. The propagable modes are computed using the resulting effective elastic material parameters from section 3 . Some propagating modes become more visible than others due to their high attenuation coefficient, especially those parts close to the cut-off frequency $(\operatorname{Re}(k)=0)$. The low frequency modes are hardly recognizable because of their low attenuation. In this depiction an attenuation close to zero means that the wave mode is either unattenuated or not excited. Therefore, Figure 2 is additionally needed for interpretation, since it shows which wave modes are detected and excited. However, Figure 4 can be used to determine the attenuation coefficient $\alpha$ of the wave modes detected in Figure 2 by searching the wave modes' frequency and real wavenumber in Figure 4. As similar information can be determined by waveguide simulations, an inverse procedure for viscoelastic material 


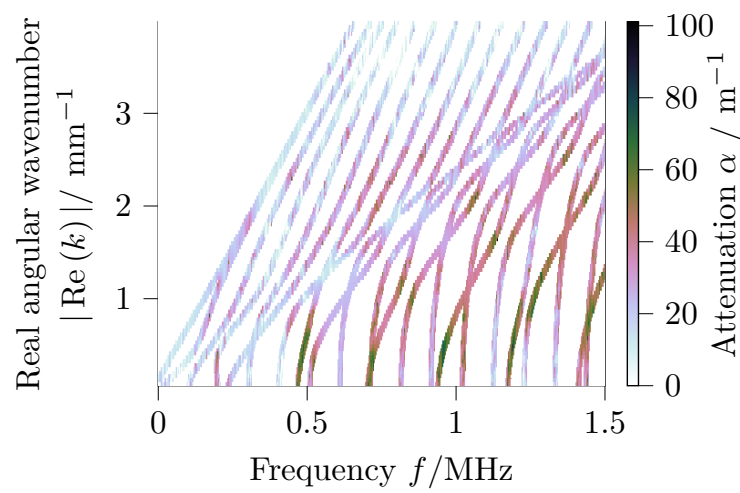

Fig. 4: Modal attenuation $\alpha$ of Lamb waves in a PEEK plate.

parameter determination can be developed based on the modal attenuation.

\section{Elastic parameter determination}

The propagating modes' frequencies and real parts of their wavenumbers (Figure 2) are used to identify an elastic material model in an inverse procedure. As the forward model for the inverse procedure a semi-analytical finite element [3] model is used to compute dispersion curves, assuming isotropic material behaviour. For the material model, the longitudinal and transversal wave velocities are identified. The plate's thickness and density $\rho$ is measured directly. As a local optimisation algorithm is used, initial values have to be estimated first.

\subsection{Initial values}

Initial values can either be determined by evaluating the propagation time in temporal and spatial domain [12], the group velocities close to the longitudinal and transversal wave velocities [9] or by evaluating the cut-off frequencies [10], which are usually hard to detect for materials with strong attenuation. Here, the second option is presented (red lines in Figure 5). For higher frequencies and wavenumbers, the first mode's group velocity converges to Rayleigh wave velocity $c_{\mathrm{R}}[2]$, which is related to transversal wave velocity with a factor close to 1 depending on the material's Poisson's ratio. As the initial value $c_{\mathrm{T}}^{(0)} \approx c_{\mathrm{R}}^{(0)}$ is assumed. The longitudinal wave velocity $c_{\mathrm{L}}^{(0)}$ is estimated in the same manner by connecting the recognizable parts of the modes with highest gradient and so the highest group velocity. Evaluating the gradients $\partial \omega / \partial \operatorname{Re}(k)$ of

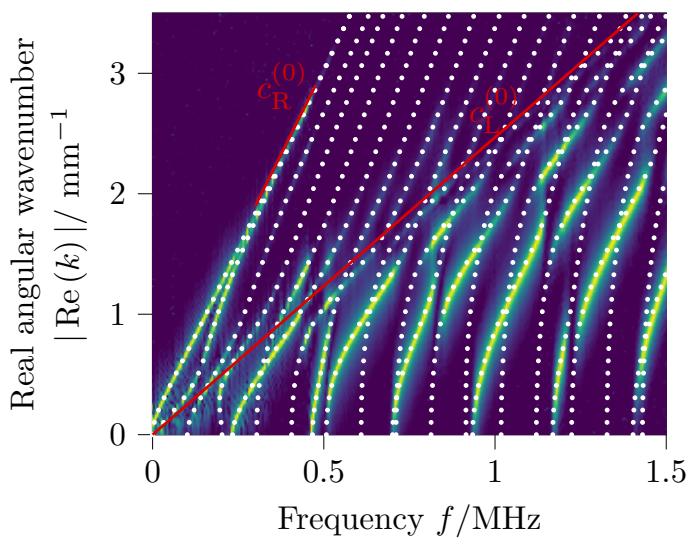

Fig. 5: Measurement data (coloured) and optimisation result for the dispersion curves (white). Red lines depict the initial value estimation for transversal $\left(c_{\mathrm{T}}^{(0)} \approx c_{\mathrm{R}}^{(0)}\right)$ and longitudinal wave velocity $\left(c_{\mathrm{L}}^{(0)}\right)$.

both lines (red) in Figure 5 yields the initial values for the wave velocities $c_{\mathrm{L}}^{(0)} \approx 2545 \mathrm{~m} \mathrm{~s}^{-1}$ and $c_{\mathrm{T}}^{(0)} \approx 1105 \mathrm{~m} \mathrm{~s}^{-1}$.

\subsection{Cost function}

For the cost function, all frequency-wavenumber pairs from the forward model are extracted from the measurement data (Figure 2). Their mean intensity value is taken as a cost function [12]. A higher mean intensity thus indicates a good match of data from measurement and forward model. Since most optimisation algorithms search for the minimum value, the negative mean intensity is used as a cost function. Note that this method does not require the application of ridge detection methods.

\subsection{Optimisation and results}

For optimisation the BOBYQA algorithm [14] is used, although every arbitrary local optimisation algorithm is expected to yield good results as the cost function is smooth [12]. For validation the depiction in frequencywavenumber domain (coloured in Figure 5) is superimposed with the computed dispersion diagram using the determined effective wave velocities (white). It shows good agreement between simulation and measurement. Using the measured plate thickness of $5.45 \mathrm{~mm}$ and the initial values for wave velocities the effective elastic wave velocities are identified for a measurement at a temperature of $20^{\circ} \mathrm{C}$ :

$$
\begin{aligned}
& c_{\mathrm{L}}=2558.28 \mathrm{~m} \mathrm{~s}^{-1}, \\
& c_{\mathrm{T}}=1109.70 \mathrm{~m} \mathrm{~s}^{-1} .
\end{aligned}
$$




\section{Viscoelastic parameter determination}

The viscoelastic material parameters are determined in an inverse procedure by analysing the real as well as the imaginary part of the wavenumber. As a material model, a modified fractional Zener model is selected.

\subsection{Modified fractional Zener model}

The acoustic damping can be modelled using complex acoustic material parameters, whereas frequency dependent models are common. A suitable model for the viscoelastic behaviour of polymers is the fractional Zener model [4]. Further, Bause [4] shows that an eigenvalue decomposition of the fractional Zener model is suitable to model different attenuation for bulk and shear components in polymers such as PEEK [4]. In general, the elasticity matrix in Voigt notation

$$
\boldsymbol{C}=\left(\begin{array}{cccccc}
K+\frac{4}{3} \mu & K-\frac{2}{3} \mu & K-\frac{2}{3} \mu & 0 & 0 & 0 \\
K-\frac{2}{3} \mu & K+\frac{4}{3} \mu & K-\frac{2}{3} \mu & 0 & 0 & 0 \\
K-\frac{2}{3} \mu & K-\frac{2}{3} \mu & K+\frac{4}{3} \mu & 0 & 0 & 0 \\
0 & 0 & 0 & \mu & 0 & 0 \\
0 & 0 & 0 & 0 & \mu & 0 \\
0 & 0 & 0 & 0 & 0 & \mu
\end{array}\right)
$$

with the bulk modulus $K$, shear modulus $\mu$ [13] can be expressed by six eigenvalues $\lambda_{i}$ and their respective eigenvectors $\boldsymbol{p}_{i}$ [16]. For an isotropic material, the eigenvalue decomposition can be simplified to three eigenvalues and eigenvectors:

$$
\boldsymbol{C}=\sum_{i=0}^{3} \lambda_{i} \boldsymbol{p}_{i}^{\mathrm{T}} \boldsymbol{p}=\sum_{i=0}^{3} \lambda_{i} \boldsymbol{D}_{i}
$$

with the eigenvalues $\lambda_{i}$, eigenvectors $\boldsymbol{p}_{i}$ and the decomposition matrix $\boldsymbol{D}_{i}=\boldsymbol{p}_{i}^{\mathrm{T}} \boldsymbol{p}_{\boldsymbol{i}}$ [4]. For the two-dimensional plane strain case the elasticity matrix and thus the matrices $\boldsymbol{D}_{i}$ reduce to $3 \times 3$, yielding the following eigenvalues

$$
\lambda_{1}=3 K, \quad \lambda_{2}=2 \mu, \quad \lambda_{3}=\mu
$$

and corresponding decomposition matrices (eigendirections):

$$
\begin{gathered}
\boldsymbol{D}_{1}=\frac{1}{3}\left(\begin{array}{lll}
1 & 1 & 0 \\
1 & 1 & 0 \\
0 & 0 & 0
\end{array}\right), \quad \boldsymbol{D}_{2}=\frac{1}{3}\left(\begin{array}{ccc}
2 & -1 & 0 \\
-1 & 2 & 0 \\
0 & 0 & 0
\end{array}\right) \\
\boldsymbol{D}_{3}=\left(\begin{array}{lll}
0 & 0 & 0 \\
0 & 0 & 0 \\
0 & 0 & 1
\end{array}\right) .
\end{gathered}
$$

Bause [4] suggests using the same damping model for the second and third eigendirection. Thus, the first eigendirection, describing pure bulk deformations, is attenuated differently than the other two eigendirections, which describe shear deformations. So the resulting complex damped elasticity matrix is given by:

$$
\begin{aligned}
\tilde{\boldsymbol{C}}= & \frac{1+d_{K}\left(\mathrm{j} \omega \tau_{\sigma_{K}}\right)^{\nu_{K}}}{1+\left(\mathrm{j} \omega \tau_{\sigma_{K}}\right)^{\nu_{K}}} 3 K \boldsymbol{D}_{1} \\
& +\frac{1+d_{\mu}\left(\mathrm{j} \omega \tau_{\sigma_{\mu}}\right)^{\nu_{\mu}}}{1+\left(\mathrm{j} \omega \tau_{\sigma_{\mu}}\right)^{\nu_{\mu}}} \mu\left(2 \boldsymbol{D}_{2}+\boldsymbol{D}_{3}\right) .
\end{aligned}
$$

Consequently, in addition to the (quasi-static) bulk and shear moduli $K$ and $\mu$, six damping parameters for modelling both bulk $(K)$ and shear $(\mu)$ deformations need to be determined. These are the time constant $\tau_{\sigma_{K / \mu}}$, the stiffness ratio $d_{K / \mu}$, as well as the fractional order of the derivative $\nu_{K / \mu}$.

\subsection{Initial values}

Similar to the elastic parameter identification, initial values are required for the local optimisation procedure. Initially, the damping parameters determined for PEEK by Bause [4] are used as initial values:

$$
\begin{array}{ll}
\tau_{\sigma_{K}}^{(0)}=0.302 \mu \mathrm{s}, & \tau_{\sigma_{\mu}}^{(0)}=0.098 \mu \mathrm{s}, \\
d_{K}^{(0)}=1.09, & d_{\mu}^{(0)}=1.079, \\
\nu_{K}^{(0)}=1, & \nu_{\mu}^{(0)}=0.4764 .
\end{array}
$$

Since the parameters $d_{K}$ and $d_{\mu}$ approximately describe the quadratic quotient of the phase velocities at infinite frequency and at quasi-static frequency the quasi-static initial wave velocities are estimated as

$$
\begin{aligned}
& c_{\mathrm{L}, 0}^{(0)} \approx \frac{c_{\mathrm{L}}}{\sqrt{d_{K}}}=2447.35 \mathrm{~m} \mathrm{~s}^{-1}, \\
& c_{\mathrm{T}, 0}^{(0)} \approx \frac{c_{\mathrm{T}}}{\sqrt{d_{\mu}}}=1068.21 \mathrm{~m} \mathrm{~s}^{-1},
\end{aligned}
$$

while the effective elastic wave velocities from Equation 2 and 3 serve as an estimate for the wave velocities at infinite frequency. The (quais-static) bulk and shear moduli are determined by the well known relations $[13,15]$ :

$$
\begin{aligned}
K^{(0)} & =\rho\left(c_{\mathrm{L}, 0}^{(0) 2}-\frac{4}{3} c_{\mathrm{T}, 0}^{(0) 2}\right) & & =5.85 \mathrm{GPa}, \\
\mu^{(0)} & =\rho c_{\mathrm{T}, 0}^{(0) 2} & & =1.49 \mathrm{GPa} .
\end{aligned}
$$

\subsection{Cost function}

To compare measurement and simulation the propagating modes are detected in preprocessed measurement data 


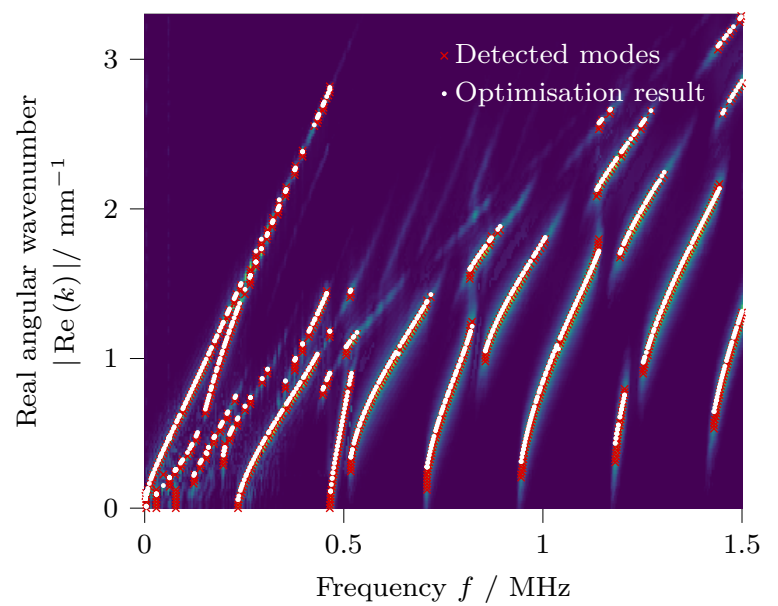

Fig. 6: Detected frequency-wavenumber pairs (white) and computed wavenumbers using the determined material parameters as input parameters (red) compared to preprocessed measurement data.

with a peak detection algorithm (red in Figure 6). This yields the frequency as well as the real part of the wavenumber of the propagating mode. To obtain the imaginary part of the wavenumber $\left(\operatorname{Im}\left(k_{\text {meas }}\right)=\alpha_{\text {mes }}\right)$, Figure 4 is evaluated at the points where modes are detected. As a cost function, the absolute linear difference of the wavenumbers' imaginary part from measurement $\operatorname{Im}\left(k_{\text {meas }}\right)$ and forward simulation $\operatorname{Im}\left(k_{\mathrm{sim}}\right)$ is used:

$$
\begin{aligned}
\epsilon= & \sum_{n}\left(|| \operatorname{Im}\left(k_{n, \text { sim }}\right)|-| \operatorname{Im}\left(k_{n, \text { meas }}\right)||\right) \\
& \cdot U_{0}\left[f_{n}, \operatorname{Re}\left(k_{n, \text { meas }}\right)\right] .
\end{aligned}
$$

The intensity $U_{0}\left[f_{\mathrm{n}}, \operatorname{Re}\left(k_{n, \text { meas }}\right)\right]$ from preprocessed data for each frequency-wavenumber pair serves as a regularisation term that reduces the influence of falsely detected frequency-wavenumber pairs. Since all wave modes for each frequency are computed, the one which real part closest to the detected one from measurement is used.

\subsection{Optimisation and results}

Using the initial parameters (subsection 4.2) and the cost function (subsection 4.3) the optimisation is performed using the detected frequency-wavenumber pairs depicted in red in Figure 6. Since the changing damping parameters also influence the wavenumbers' real part, an additional optimisation of the (quasi-static) wave velocities is performed afterwards while now assuming a viscoelastic material model. Again, the BOBYQA-algorithm [14] is used. The frequency-wavenumber pairs detected and considered for the optimisation process are shown in red in Figure 6 which are mostly overlaid by the frequency-wavenumber pairs computed using the optimised viscoelastic material parameters (white). Note that only the previously detected frequency-wavenumber pairs are depicted. Figure 7 shows the modal attenuation $|\alpha|$ for each detected mode from measurement (blue), the attenuation computed using the initial values (red) and the attenuation computed using the determined optimised parameters (black). For small frequencies up to $200 \mathrm{kHz}$ artefacts, e.g. the leakage effect, lead to detected frequency-wavenumber pairs, which do not belong to any wave mode (Figure 6 and 7 ). While the attenuation computed using the initial values shows some deviations from the measurement, the attenuation computed using the optimised parameters matches the measured attenuation well in the range above $0.5 \mathrm{MHz}$. The remaining deviation may be further reduced by applying a revised optimisation strategy.

For PEEK at a temperature of $20^{\circ} \mathrm{C}$, the following viscoelastic material parameters are determined:

$$
\begin{array}{rlrl}
K & =6.24 \mathrm{GPa}, & \mu & =1.54 \mathrm{GPa}, \\
c_{\mathrm{L}} & =2517.12 \mathrm{~m} \mathrm{~s}^{-1}, \quad c_{\mathrm{T}} & =1084.74 \mathrm{~m} \mathrm{~s}^{-1}, \\
\tau_{\sigma_{K}} & =0.293 \mu \mathrm{s}, & \tau_{\sigma_{\mu}} & =0.096 \mu \mathrm{s}, \\
d_{K} & =1.022, & d_{\mu} & =1.131, \\
\nu_{K} & =0.719, & \nu_{\mu} & =0.504 .
\end{array}
$$

The determined quasi-static wave velocities are close to the effective wave velocities obtained from elastic parameter determination because PEEK generally shows low attenuation compared to other polymers as e.g. polyamides. The identified parameters for the fractional Zener model are similar to the ones determined by Bause [4], however, as Figure 7 shows, the resulting values for the modal attenuation show a better match with the measurement. The deviations may be explained by differences in the manufacturing processes for the hollow cylinders analysed by Bause and the plates used in this study.

\section{Conclusions}

Using broadband acoustic plate waves, the elastic and viscoelastic material parameters of a polymeric sample are determined in an inverse procedure. The approach is based on spatio-temporal measurement data that is processed in a new method to determine the real and the imaginary part of the wavenumber of the propagating modes.

Further research will include the development of an estimator for the initial values, which, as of now, relies on literature. Based on a sensitivity study the optimisation procedure will be adapted and alternative cost functions 


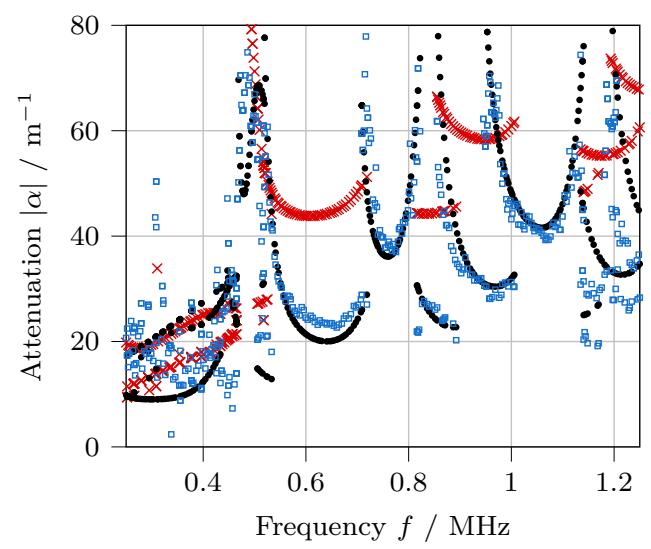

Fig. 7: Measured modal attenuation ( $(\mathrm{)})$ and simulated attenuation using the inital estimate $(\times)$ and the optimised material parameters $(\cdot)$.

e.g. using the absolute value of the wavenumbers will be evaluated. Finally, the authors aim to apply the method to different polymeric samples and other solids, evaluating the possibility of applying alternative material models. Long term goals include the development of a material characterisation method based on the presented approach that is applicable in industrial environments.

Acknowledgment: The authors would like to thank the staff at Paderborn Center for Parallel Computing $\left(\mathrm{PC}^{2}\right)$ for supplying access to the OCuLUS cluster and the German Research Foundation (DFG) for financial support of the project no. 449607253 .

\section{References}

[1] D. Alleyne and P. Cawley. A 2-dimensional Fourier transform method for the quantitative measurement of Lamb modes. In IEEE Symposium on Ultrasonics, pages 1143-1146. IEEE, 1990. 10.1109/ULTSYM.1990.171541.

[2] B. A. Auld. Acoustic fields and waves in solids, volume 1. Krieger Publishing Company, Malabar, Florida, 2 edition, 1990. ISBN 0-89874-782-1.

[3] I. Bartoli, A. Marzani, F. Di Lanza Scalea, and E. Viola. Modeling wave propagation in damped waveguides of arbitrary cross-section. Journal of Sound and Vibration, 295(3-5): 685-707, 2006. ISSN 0022460X. 10.1016/j.jsv.2006.01.021.

[4] F. Bause, J. Rautenberg, N. Feldmann, M. Webersen, L. Claes, H. Gravenkamp, and B. Henning. Ultrasonic transmission measurements in the characterization of viscoelasticity utilizing polymeric waveguides. Measurement Science and Technology, 27(10), 2016. 10.1088/09570233/27/10/105601.
[5] A. G. Bell. Upon the production and reproduction of sound by light. Journal of the Society of Telegraph Engineers, 9(34): 404-426, 1880. ISSN 2054-0698. 10.1049/jste-1.1880.0046.

[6] C. Bermes, J.-Y. Kim, J. Qu, and L. J. Jacobs. Experimental characterization of material nonlinearity using Lamb waves. Applied Physics Letters, 90(2):021901, Jan. 2007. $10.1063 / 1.2431467$.

[7] N. Bochud, J. Laurent, F. Bruno, D. Royer, and C. Prada. Towards real-time assessment of anisotropic plate properties using elastic guided waves. Journal of the Acoustical Society of America, 143:1138-1147, 2018.

[8] L. Claes, T. Meyer, F. Bause, J. Rautenberg, and B. Henning. Determination of the material properties of polymers using laser-generated broadband ultrasound. Journal of Sensors and Sensor Systems, 5(1):187-196, 2016. ISSN 2194-878X. 10.5194/jsss-5-187-2016.

[9] L. Claes, A. Jäger, S. Johannesmann, M. Webersen, M. Kupnik, and B. Henning. Acoustic material characterization of additively manufactured components. In AMA Service $\mathrm{GmbH}$, editor, PROCEEDINGS - AMA Conferences 2017, pages 605-610, 2017. ISBN 978-3-9816876-4-4. 10.5162/sensor2017/P2.9.

[10] L. Claes, H. Schmiegel, C. Grünsteidl, S. Johannesmann, M. Webersen, and B. Henning. Investigating peculiarities of piezoelectric detection methods for acoustic plate waves in material characterisation applications. tm - Technisches Messen, 88(3), 2021. ISSN 0171-8096.

[11] C. Grünsteidl, T. Berer, M. Hettich, and I. Veres. Determination of thickness and bulk sound velocities of isotropic plates using zero-group-velocity Lamb waves. Applied Physics Letters, 112, 06 2018. 10.1063/1.5034313.

[12] S. Johannesmann, L. Claes, M. Webersen, and B. Henning. Inverser Ansatz zur akustischen Charakterisierung plattenförmiger Materialproben. In Deutsche Gesellschaft für Akustik e.V. 2017- Fortschritte der Akustik // Fortschritte der Akustik - DAGA 2017, pages 999-1002, Berlin, 2017. Deutsche Gesellschaft für Akustik e.V. (DEGA). ISBN 9783939296126.

[13] F. P. Mechel and M. L. Munjal. Formulas of acoustics. Springer, Berlin, 2 edition, 2008. ISBN 3540768327.

[14] M. Powell. The BOBYQA algorithm for bound constrained optimization without derivatives. Technical Report, Department of Applied Mathematics and Theoretical Physics, 01 2009.

[15] J. Rosenbaum. Bulk Acoustic Wave Theory and Devices. Acoustics and Signal Processing Library. ARTECH HOUSE Incorporated, 1988. ISBN 9780890062654.

[16] P. S. Theocaris and D. P. Sokolis. Spectral decomposition of the compliance tensor for anisotropic plates. Journal of Elasticity, 51(2):89-103, 1998. ISSN 03743535. 10.1023/A:1007549729716. 